\title{
Analysis of Student Feedback using Deep Learning
}

\author{
Shejwal Asmita $S$ \\ Jaihind College Of \\ Engineering, Kuran, \\ Pune
}

\author{
Deokar Anuja T \\ Jaihind College Of \\ Engineering, Kuran, \\ Pune
}

\author{
Dumbre Ashwini B. \\ Jaihind College Of \\ Engineering, Kuran, \\ Pune
}

\begin{abstract}
Feedback plays a key role in improving quality. To ensure improvement in teaching method and facilities provided by college, opinion of the students should be properly analysed and used. Text Sentiment analysis method are used to carry out such analysis. It can be performed in two ways - Machine Learning approach and Lexicon based approach. Presently, the teacher evaluation and feedback analysis are based on identifying student's opinion. Methods used for such classification are Naive Bayes, Voting ensemble method. Along with determining polarity, classifying feedback as strength, weakness and suggestions can improve to be more beneficial. Success of deep learning inspires us to propose a better and efficient system. The System that will use Word2Vec for text processing, Convolution Neural Network for automatic feature extraction. Supervised Support Vector Machine will be used for final classification. The Proposed system will result in classification of feedback as Strength, Weakness and Suggestions to faculty.
\end{abstract}

\section{Keywords: Supervised learning, Convolution neural network, feature extraction, Sentiment Analysis, Deep learning.}

\section{INTRODUCTION}

Youth is the hope and future of nations. Today's youth are students. Students play a vital role in society. It is primary necessity that education given to students needs to be quality education and must consider their views regarding it. Technology has allowed students to explore new fields. Hence considering student point of view to improve educational system can be beneficial. To keep track of performance and help improve abilities of faculty, opinions of students can be helpful. Finding out the subjective meaning from opinions is the major task. Sentiment analysis can be one way to do it. Sentiment analysis is contextual mining of text which identifies and extracts subjective information in source material, and helps to understand the social sentiment when monitoring an organization. Natural language processing (NLP) have to do with building of computational algorithms to automatically analyze and represent human language. The branch of natural language processing is shifting from statistical methods to neural network methods. Deep learning methods are achieving state-of-the-art results on challenging machine learning problems for example describing photos and translating text from one language to another. Our proposed system will be useful in educational sector. The feedback of students, collected in the form of text, will be analyzed using word2vec and CNN. The proposed system will result in classification of feedback as strength, weakness and suggestions to faculty.

\section{LITERATURE SURVEY}

Most existing sentiment analysis algorithms were designed for binary classification, meaning that they assign opinions or reviews to bipolar classes such as Positive or Negative. A series of experiments with convolutional neural networks built on top of word2vec are described in [2]. The results of experiment show that unsupervised pre-training of word vectors is an important ingredient in deep learning for
NLP. In [7] the paragraph of sentences given by the customer is accepted and after extracting each and every word, they are checked with the stored parts of speech, articles and negative words. After checking against the database, Context free Grammar (CFG) is used to validate proper formation of the sentences.

In [3] Automatic evaluation system based on sentiments to overcome drawback of traditional questionnaire system. Feedback is collected in the form of running text and sentiment analysis is performed to identify important aspects along with the orientations using supervised and semi supervised machine learning techniques. It focuses more on subjective sentences and not on objective sentences. The scores are collected and aggregated to calculate final result. Term Frequency - Inverse Document Frequency (TF-IDF) and Naïve Bayes (Unigram, Bigrams) methods is used. It does not use advanced machine learning techniques and so the results were not accurate. Author of [4] states Text Mining techniques are broadly extended to classify the effective improvement of sentiment polarity analysis. Different techniques like Support Vector Machine (SVM), KNN and Decision tree are generally used but they are not always effective. Reducing the feature in data pre-processing stage and teaching sentiment analysis using voting ensemble method of machine learning are proposed and compared with existing typical machine learning for sentiment analysis.

The system achieves accuracy improvement of subjective polarity in sentiment analysis. Lack of weight assignment for feature extraction is observed. Methods like Naïve Bayes, ID3, J48 Decision Tree are used. The system described in [6] evaluates faculty and rates them with certain specified parameter to improve academic and education standard. The system is based on attribute and uses multipoint rating system. System uses text mining for deriving high quality information. Academic performance of students is considered when using the feedback given by them. Weights are assigned to feedback based on academic performance and sincerity. Multipoint rating is provided. The number of comparisons can be reduced in the system for more effective system. Naive Bayes method is used for text mining. 
In [1] Pre-trained Word2Vec for text pre-processing and to gain vector representations of words which will be the input for suitable Convolutional Neural Network (CNN) architecture for deep features extraction is applied. Rectified Linear Unit and Dropout functions is used to improve the accuracy. Support Vector Machine classifier was used to predict the final classification. Author of [9] combines the advantages of CNNs and SVM, and constructs a text sentiment analysis model based on CNNs and SVM. The pretrained word vector is used as input, and CNNs is used as an automatic feature learner, and SVM is the final text classifier. It is found that the accuracy of using CNN model results in better other models of depth learning, which shows that CNN model is more suitable to deal with text affective classification problem [8].

\section{ARCHITECTURE}

Students will give the feedback in the form of text and opinions regarding the faculty members and facilities provided by educational institute. The system will make use of Word2Vec for text processing. In word2vec, a distributed representation of a word is used. Take a vector with several hundred dimensions (say 1000). Each word is represented by a distribution of weights across those elements. So instead of a one-to-one mapping between an element in the vector and a word, the representation of a word is spread across all of the elements in the vector, and each element in the vector contributes to the definition of many words. Convolution Neural Network will the perform automatic feature extraction. Supervised Support Vector Machine will be used for final classification. Finally, the faculties will be notified about their strength and weakness and suggestion given by students [11].

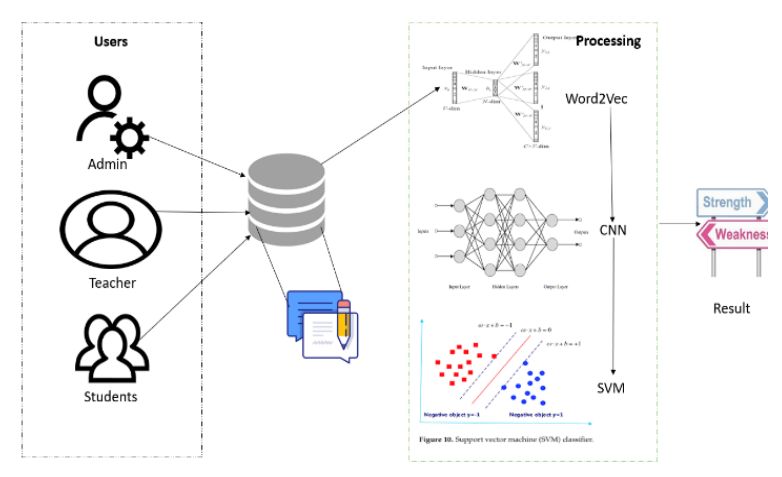

Figure 1. System Architecture

\subsection{Word2Vec}

Humans are good at alphanumeric but to process and analyse the huge amount of text devices like computer (processing unit) is necessary. But computers cannot understand human language. In order to process, sentiments they must be converted to numeric form. Word2vec is a particular machine learning model that produces word embedding. Word Embedding mean a word can be associated to a number. One can perform "math" with numeric values to find similarity between words. In neural network or word embeddings we define a model that aims to predict between a centre word Wt and Context words in terms of Word Vectors
The idea behind Word2vec is to predict words and its context word. The Word2Vec is based on two algorithms Skip gram and Continuous Bag of Words (CBOW). It is represented as $\mathrm{P}($ context $\mid \mathrm{Wt})$. The Skip gram predict context words given a target word whereas CBOW predict target word from bag of word context[10].

\subsection{Convolutional Neural Network}

$\mathrm{CNN}$ takes input data then performs convolutions on the data and apply pooling to data. To better understand what actually the CNN does consider a vector for sentence given as input to $\mathrm{CNN}$. The vector is output of the word2vec text processing. A binary operation is performed to extract feature. Output of word $2 \mathrm{vec}$ is first operand for operation and filter second. The sequence generated after operation is the feature map. The convolution process can be explained using diagrammatic representation as shown below. Example: "He is experienced and knowledgeable.". The vector for given sentence is considered as input to the CNN model. A 2-word filter is applied to the sentence[9].

Step 1: The filter convolves over the first to words and feature map value is calculates. That is if words in sentence are $\mathrm{Wt}, \mathrm{Wt}+1 \ldots \ldots . \mathrm{WT}$. The convolution operation is carried out on $\mathrm{Wt}, \mathrm{Wt}+1$ as shown in figure.

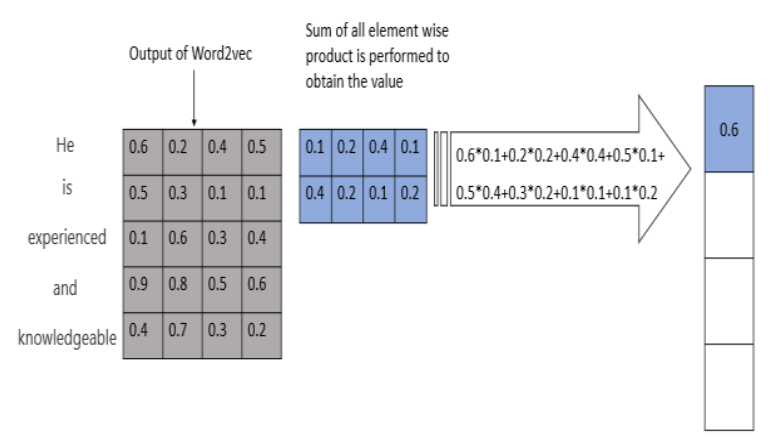

Figure 2. CNN Example step 1

Step2: In second step the sliding window shifts on place forward and calculate the feature map value for next two words i.e. $\mathrm{Wt}+1$ and the next word.

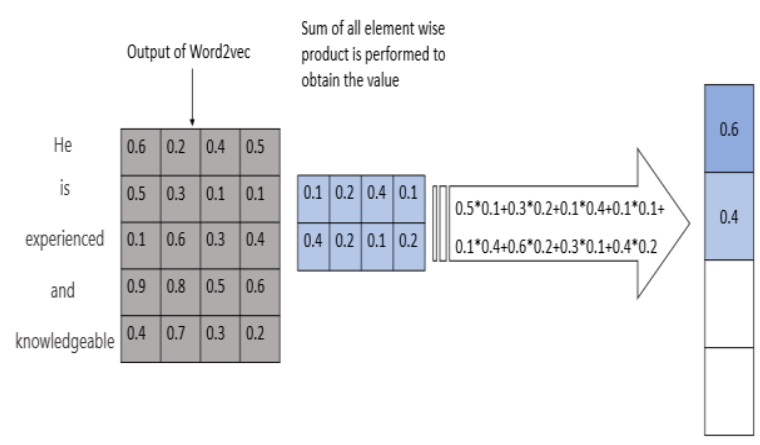

Figure 3. CNN Example step 2 
The process continues till the last two words. The feature map for "He is experienced and knowledgeable" is shown in figure

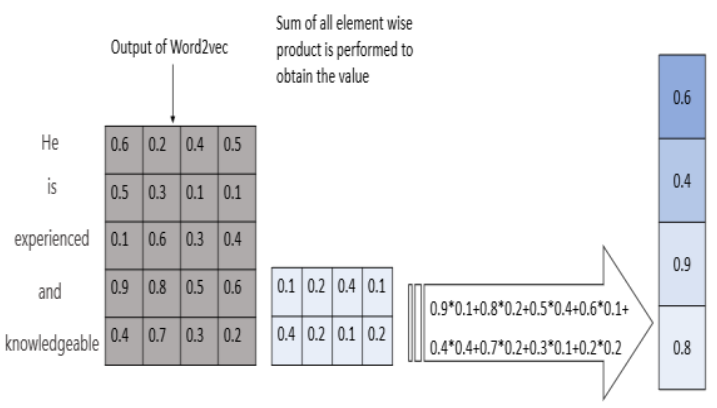

Figure 4. CNN Example step 1

Layer for automatically features extraction using three convolution kernels (convolution filters) of different sizes, ReLu Layer, Pooling Layer with nonlinear sampling method in order to decrease the number of characteristic parameters and prevent overfitting, and Fully-Connected Layer[2].

\subsection{Support Vector Machine}

SVM is a type of classification algorithm which classifies data based on its feature. It is type of binary classifier. Convolution neural network can extract meaningful feature representation from input samples effectively, but the classification ability of fully connected classification layer is weak for nonlinear separable data. SVM is a supervised machine learning model, which is two-classification model. The SVM method is based on the theory of statistical learning theory and the principle of minimum structural risk. According to the limited data information, SVM try to find the best compromise between the complexity and the learning ability, in order to get the best generalization ability. SVM can find the optimal classification surface for the characteristics. As shown in figure it considers the best line of separation between data points. In figure the blue circles represent strengths and orange block represent weakness. When a new point is detected it is checked across hyperplane of SVM. The hyperplane determines whether it is strength and weakness[12].

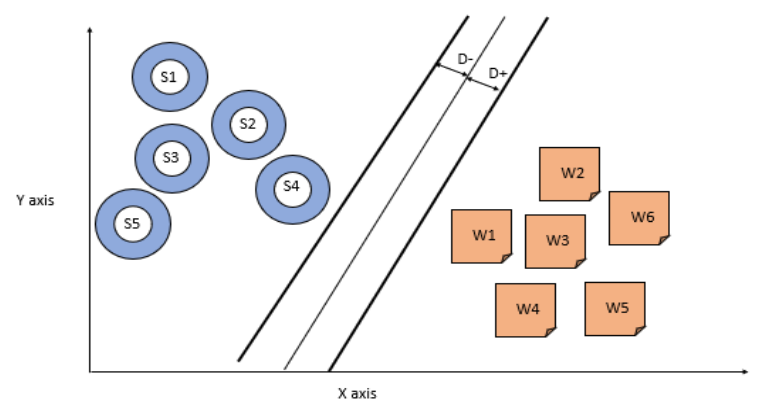

Figure 5. SVM Classification

\section{MATHMATICAL MODEL} System Description:

Let $\mathrm{S}$ be a closed graph system that draws the object; such that $\mathrm{S}=\mathrm{I}, \mathrm{P}, \mathrm{R}, \mathrm{O}$ ?s where I represents the set of Input; $\mathrm{I}=\mathrm{i} 0$ ? $\mathrm{i}$ and $\mathrm{P}$ is a set of Process; $\mathrm{P}=$ p0,p1,p2,?p and $\mathrm{R}$ is set of rules $\mathrm{R}=\mathrm{r} 0, \mathrm{r} 1$ and $\mathrm{O}$ is set of Outputs; $\mathrm{O}=\mathrm{o} 0, \mathrm{o} 1$

Where,

- $\quad$ I=Input

$\mathrm{I}=\mathrm{I} 1$

Where,

I1 = Student Feedback

- $\quad \mathrm{P}=$ Process

$\mathrm{P}=\mathrm{P} 1, \mathrm{P} 2, \mathrm{P} 3$

$\mathrm{P} 1=$ Student Process

P11= Student Registration

P12= Student Login

$\mathrm{P} 13=$ For particular subject i.e. for respective teacher Fill Feedback Form

P14= logout

- $\quad \mathrm{P} 2=$ Admin Process

P21 = Admin Login

P22= Manage Feedback

P23 = Manage User and Full Application

P24= Analysis Feedback Report

P25= Analysis Faculty Report

- $\quad \mathrm{P} 26=$ Logout

P3= Teacher Process

P31 = Teacher login

P32 $=$ View Feedback (Strength $/$

Weakness/ Suggestions)

P33= View Ranking

- $\quad \mathrm{R}=$ rules

R1=Login Validations

R2 = Validate type of Access (Student/ Admin/ Faculty).

- $\mathrm{O}=$ Output

O1 $=$ Generate Feedback Type report

$\mathrm{O} 2=$ Generate Class Report

Activity 1 Let fi be a rule of $\mathrm{I}$ into $\mathrm{P}$ such that student will feed the data into the system. it returns fI(i0) i p0,p1, p2 P. 
Activity2 Let fp be a rule of $\mathrm{P}$ into $\mathrm{R}$ such that the processed data will go through particular

set of rules it returns

$\mathrm{fp}(\mathrm{p} 0)$ ¿ r0,r1 R.

Activity3 Let fr be a rule of $\mathrm{R}$ into $\mathrm{O}$ such that the processed data will give the respective

output. it returns

for $(\mathrm{R} 0)$ i $\mathrm{O} 0, \mathrm{O} 1 \mathrm{O}$. As described in activity 1 following vein diagram is drawn: (Analysis: The system will give the result of feedback based on the data feed by the registered student. The student will select the respective subject/teacher then will answer respective questions and also give suggestion. The process will analysis this feeds and generate the results. Admin and teacher will be able to see the response of the students. Text of State Diagram: State Diagram of the system is shown below: Where,

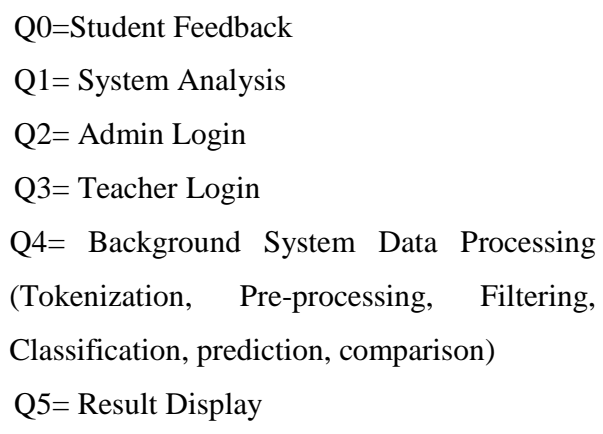

\section{RESULT AND CONCLUSION}

Students Feedback analysis using deep learning can be useful in educational sector. The expected result is a classification of the students feedback in strength and weakness of teachers.

\section{ACKNOWLEDGMENTS}

We are thankful to Jaihind College of Engineering for providing the resources. We are also thankful to Prof. Khatri A.A, project co-ordinator, and Prof. Gore S.S, Guide, for continuous support.

\section{REFERENCES}

[1] A bubakr H. Om babi, O nsaLazeez, Wael Ouarda, Adel M. Alimi "Deep Learning Framework based on Word2Vec and CNN for Users Interests Classification" 978-1-5386-0667-4/17/\$31.00 (c)2017.

[2] Yoon Kim "Convolutional Neural Networks for Sentence Classification" Proceedings of the 2014 Conference on Empirical Methods in Natural Language Processing (EMNLP), pages 17461751,October 25-29, 2014, Doha, Qatar. (02014.

[3] Alok Kumar, Renu Jain "Sentiment Analysis and Feedback Evaluation" 978-1-4673-67479/15/\$31.002015.

[4] Chakrit Pong-Inwong, KonpusitKaewmak "Improved Sentiment Analysis for Teaching Evaluation Using Feature Selection and Voting Ensemble Learning Integration" 978-1-4673-90262/16/\$31.00 @2016.

[5] Tanzim Mahmud, K. M. Azharul Hasan, Mahtab Ahmed, Thwoi Hla Ching Chak "A Rule Based Approch for NLP Based Query Processing" 978-14673-9257-0/15/\$31.00 (C2015 IEEE.

[6] Krishnaveni K S, Rohit R Pai, Vignesh Iyer "Faculty Rating System Based on Student Feedbacks Using Sentimental Analysis" 978-15090-6367-3/17/\$31.00 @2017.

[7] Biswarup Nandi, MousumiGhanti, Souvik Paul "TEXT BASED SENTIMENT ANALYSIS" 978-15386-4031-9/17/\$31.00 @2017.

[8] Fan Xia, Zhi Zhang "Study of Text Emotion Analysis Based on Deep Learning"978-1-53863758-6/18/\$31.00 c 2018.

[9] Yuling Chen, Zhi Zhang "Research on text sentiment analysis based on CNNs and SVM" 978$1-5386-3758-6 / 18 / \$ 31.00$ c 2018.

[10] Tomas Mikolov, Kai Chen, Greg Corrado, and Je rey Dean. E cient "Estimation of word representations in vector space." 012013.

[11] Shejwal Asmita S, Deokar Anuja T, Dumbre Ashwini B, Prof.S.S.Gore, "REVIEW ON STUDENT FEEDBACK ANAYSIS USING DEEP LEARNING” IJRAR1904692 (C) 2018 IJRAR November 2018, Volume 5, Issue 4.

[12] Walaa Medhat a, *, Ahmed Hassan b, Hoda Korashy b " Sentiment Analysis Algorithms and applications: A survey " Ain Shams Engineering Journal (2014) 5, 1093-1113 